\section{International Scientific Journal}

\section{Theoretical \& Applied Science}

\author{
p-ISSN: 2308-4944 (print) e-ISSN: 2409-0085 (online) \\ Year: $2016 \quad$ Issue: 9 Volume: 41 \\ Published: $30.09 .2016 \quad$ http://T-Science.org
}

SECTION 4. Computer science, computer engineering and automation.
L. M. Nabiulina

Ph.D., Manager to department. Tashkent State Pedagogical University, Tashkent, Republic of Uzbekistan qushqor@mail.ru

Sh. Abdurakhmanova Teacher, Tashkent State Pedagogical University, Tashkent, Uzbekistan

\title{
SOFTWARE MICROSOFT EXCEL FOR STATISTICAL CALCULATIONS PEDAGOGICAL EXPERIMENT BASED ON THE CRITERIA $\chi 2$ (CHI-SQUARE)
}

\author{
Abstract: The article deals the basics of processing and presentation of experimental data in Microsoft Excel \\ through the use of the criterion $\chi^{2}$ (chi-square). \\ Key words: statistical hypothesis, null hypothesis, alternative hypothesis, criterion $\chi 2$ (Chi-square). \\ Language: Russian \\ Citation: Nabiulina LM, Abdurakhmanova S (2016) SOFTWARE MICROSOFT EXCEL FOR \\ STATISTICAL CALCULATIONS PEDAGOGICAL EXPERIMENT BASED ON THE CRITERIA $\chi^{2}$ (CHI- \\ SQUARE). ISJ Theoretical \& Applied Science, 09 (41): 114-123. \\ Soi: http://s-o-i.org/1.1/TAS-09-41-17 Doi: crossef http://dx.doi.org/10.15863/TAS.2016.09.41.17

\section{ИСПОЛЬЗОВАНИЕ ПРОГРАММЫ МІСRОSОFТ ЕХСЕL ДЛЯ ПРОВЕДЕНИЯ СТАТИСТИЧЕСКИХ РАСЧЕТОВ ПЕДАГОГИЧЕСКОГО ЭКСПЕРИМЕНТА НА ОСНОВЕ КРИТЕРИЯ $\chi^{2}$ (ХИ-КВАДРАТ)}

Аннотация: В статье рассматриваются основы обработки и представления экспериментальных данных в программе Microsoft Excel на основе использования критерия ұ2 (хи-квадрат).

Ключевые слова: статистическая гипотеза, нулевая гипотеза, альтернативная гипотеза, критерий $\chi^{2}$ (хи-квадрат).

\section{Introduction}

Сегодня мы наблюдаем все более широкое внедрение информационных технологий во все сферы деятельности человека: бизнес, управление, развлечение, в том числе и образование - это дистанционное обучение, разработка электронных образовательных ресурсов, поиск информации в сети для учебного процесса, проведение телеконференций и др. Однако применению компьютерных технологий в педагогических исследованиях уделено недостаточно внимания, хотя они являются необходимым и важным инструментом.[1-4]

Для обработки количественных данных, полученных в ходе анкетирования, тестирования, ранжирования, регистрации, социометрии, интервью, беседы, наблюдений и педагогического эксперимента часто применяются математические

методы исследования с использованием компьютера. Применение методов математической или статистической обработки информации педагогических исследований требует проведения большого числа простых арифметических операций над большим числом исходных данных, занимающих много времени и неизбежно содержат множество ошибок. Сократить трудоёмкость расчётов и уменьшить число ошибок помогает использование персональных компьютеров.[5-8]

Сложные статистические расчёты поводятся с помощью специальных программ для статистических расчётов, например программа STADIA, STATISTICA, StatGraphics и SPSS. Однако данные программы, во-первых, являются лицензионными и стоят достаточно дорого. Вовторых, они достаточно сложны и требуют значительных временных затрат для своего освоения. Наряду с этим, существуют инструменты статистического анализа в электронных таблицах Microsoft Excel, входящих 


\begin{tabular}{|c|c|c|c|c|c|c|}
\hline Impact Factor: & $\begin{array}{l}\text { ISRA (India) } \\
\text { ISI (Dubai, UAE } \\
\text { GIF (Australia) } \\
\text { JIF }\end{array}$ & $\begin{array}{r}=1.344 \\
=0.829 \\
=0.564 \\
=1.500\end{array}$ & $\begin{array}{l}\text { SIS (USA) } \\
\text { PUHЦ (Russia } \\
\text { ESJI (KZ) } \\
\text { SJIF (Morocce }\end{array}$ & $\begin{array}{l}=0.912 \\
=0.234 \\
=1.042 \\
=\mathbf{2 . 0 3 1}\end{array}$ & $\begin{array}{l}\text { ICV (Poland) } \\
\text { PIF (India) } \\
\text { IBI (India) }\end{array}$ & $\begin{array}{l}=6.630 \\
=1.940 \\
=4.260\end{array}$ \\
\hline
\end{tabular}

в стандартный комплект Microsoft Office. Microsoft Excel позволяет заносить данные исследования в электронные таблицы, создавать формулы, сортировать, фильтровать, группировать данные, проводить быстрые вычисления на листе таблицы, используя «Мастер функций». С табличными данными также можно проводить статистические операции, если к Microsoft Excel подключён пакет анализа данных.

Табличный редактор Microsoft Excel c помощью встроенного мастера диаграмм также даёт возможность построить на основании результатов статистической обработки данных различные графики и гистограммы, которые можно впоследствии использовать на других этапах исследования.

\section{Materials and Methods}

Приведем пример реализации в программе Microsoft Excel обработки экспериментальных данных педагогического эксперимента с помощью метода хи-квадрат.

Предположим, что в ходе педагогического эксперимента проверялась гипотеза о влиянии разработанных лабораторных работ по курсу «Информатика и ИТ» на повышение уровня знаний и умений обучаемых. Эксперимент состоит в проведении занятий по курсу «Информатика и ИТ» на основе предложенной исследователем новой методики в экспериментальной группе, которое сравнивается c контрольной группой, обучаемой по традиционной методике. [10]

Таблица 1.

\section{Количество студентов экспериментальной и контрольной групп}

\begin{tabular}{|l|c|c|c|}
\hline & $\begin{array}{c}2013-2014 \\
\text { учебный год }\end{array}$ & $\begin{array}{c}2014-2015 \\
\text { учебный год }\end{array}$ & Всего \\
\hline Экспериментальная группа & 54 & 57 & 111 \\
\hline Контрольная группа & 51 & 53 & 104 \\
\hline
\end{tabular}

При проведении эксперимента необходимо учитывать требование репрезентативности при подборе экспериментальных и контрольных групп. Поэтому перед проведением эксперимента необходимо провести диагностическое занятие, в ходе которого экспериментальной и контрольной группам будет предложено выполнение определенных заданий. Результаты контрольного среза оформляются в виде таблицы, а количество правильных ответов в процентах в виде полигона частот.

Предположим, что в ходе диагностирования вы дали 4 задания. Разместим результаты проверочной работы в виде следующей таблицы MS Excel :

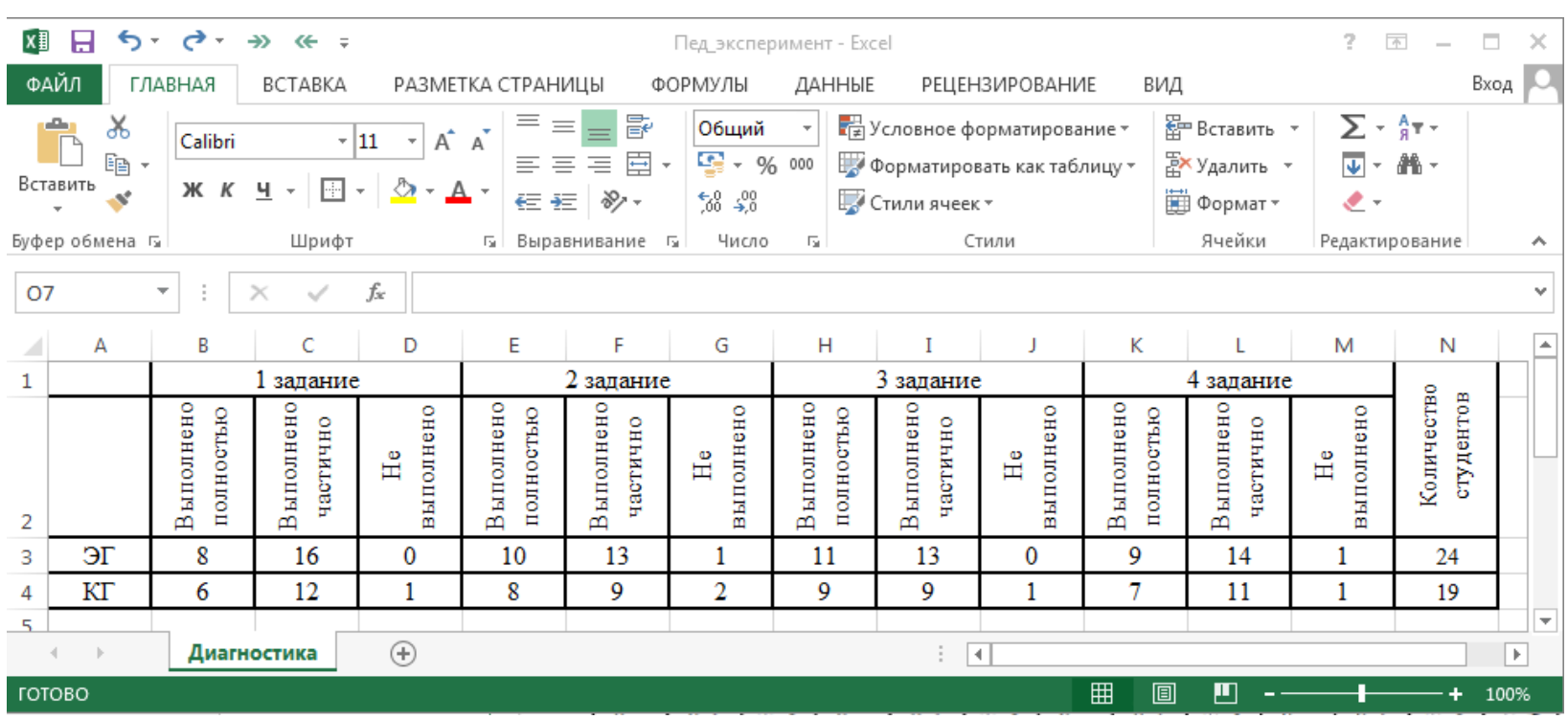

Рисунок 1 - Результаты диагностической контрольной работы.

ISPC Global Applied Research,

Harrisburg, USA 


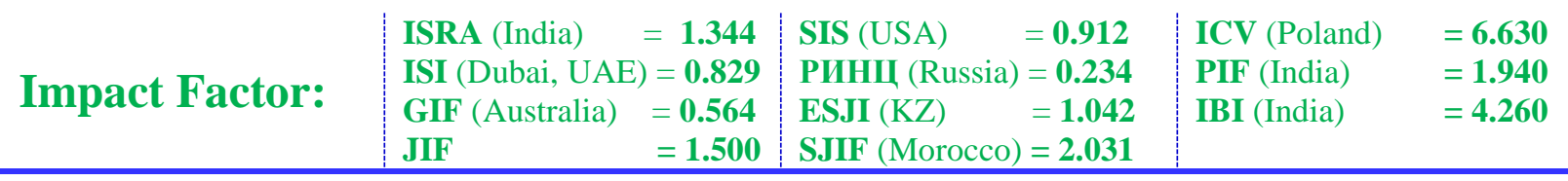

Далее необходимо рассчитать в процентах количество выполненных правильно работ: создаем таблицу, в которой необходимо ввести формулы и установить процентный формат:

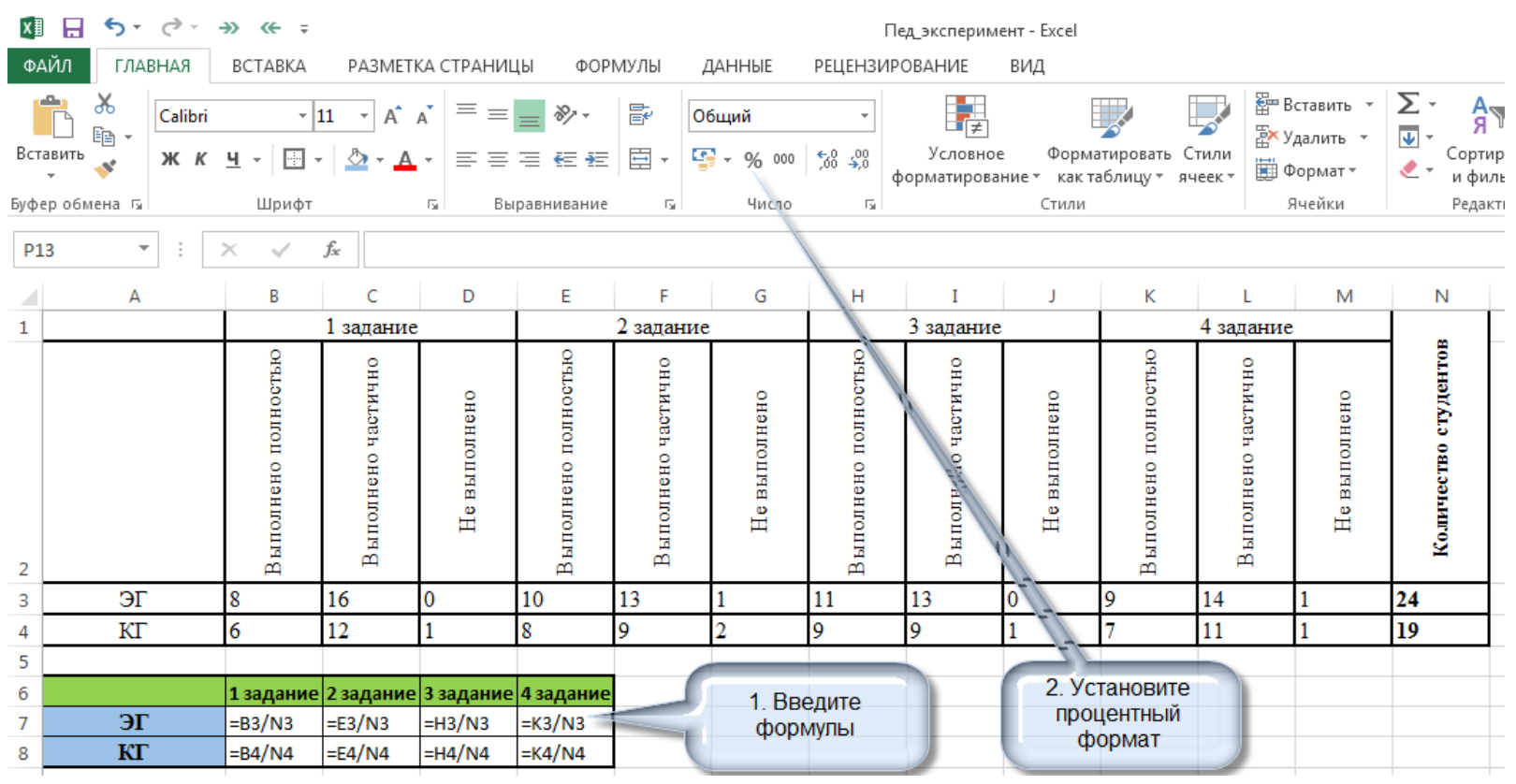

Рисунок 2 - Вычисление результатов в процентном соотношении.

В итоге у вас получится следующая таблица:

\begin{tabular}{|c|c|c|c|c|}
\hline & 1 задание & 2 задание & 3 задание & 4 задание \\
\hline ЭГ & $33 \%$ & $42 \%$ & $46 \%$ & $38 \%$ \\
\hline $\mathbf{K \Gamma}$ & $32 \%$ & $42 \%$ & $47 \%$ & $37 \%$ \\
\hline
\end{tabular}

Для получения полигона частот необходимо выделить последнюю таблицу и выполнить следующие команды: на вкладке Вставка в группе Диаграмма выбрать Точечная с гладкими кривыми и маркерами: 


\begin{tabular}{l|lrl|l|ll} 
& ISRA (India) & $=\mathbf{1 . 3 4 4}$ & SIS (USA) & $=\mathbf{0 . 9 1 2}$ & ICV (Poland) & $=\mathbf{6 . 6 3 0}$ \\
Impact Factor: & ISI (Dubai, UAE) $=\mathbf{0 . 8 2 9}$ & PUHL (Russia) $=\mathbf{0 . 2 3 4}$ & PIF (India) & $=\mathbf{1 . 9 4 0}$ \\
& GIF (Australia) & $\mathbf{0 . 5 6 4}$ & ESJI (KZ) & $=\mathbf{1 . 0 4 2}$ & IBI (India) & $=\mathbf{4 . 2 6 0}$ \\
& JIF & $\mathbf{1 . 5 0 0}$ & SJIF (Morocco) & $=\mathbf{2 . 0 3 1}$ & & \\
\hline
\end{tabular}

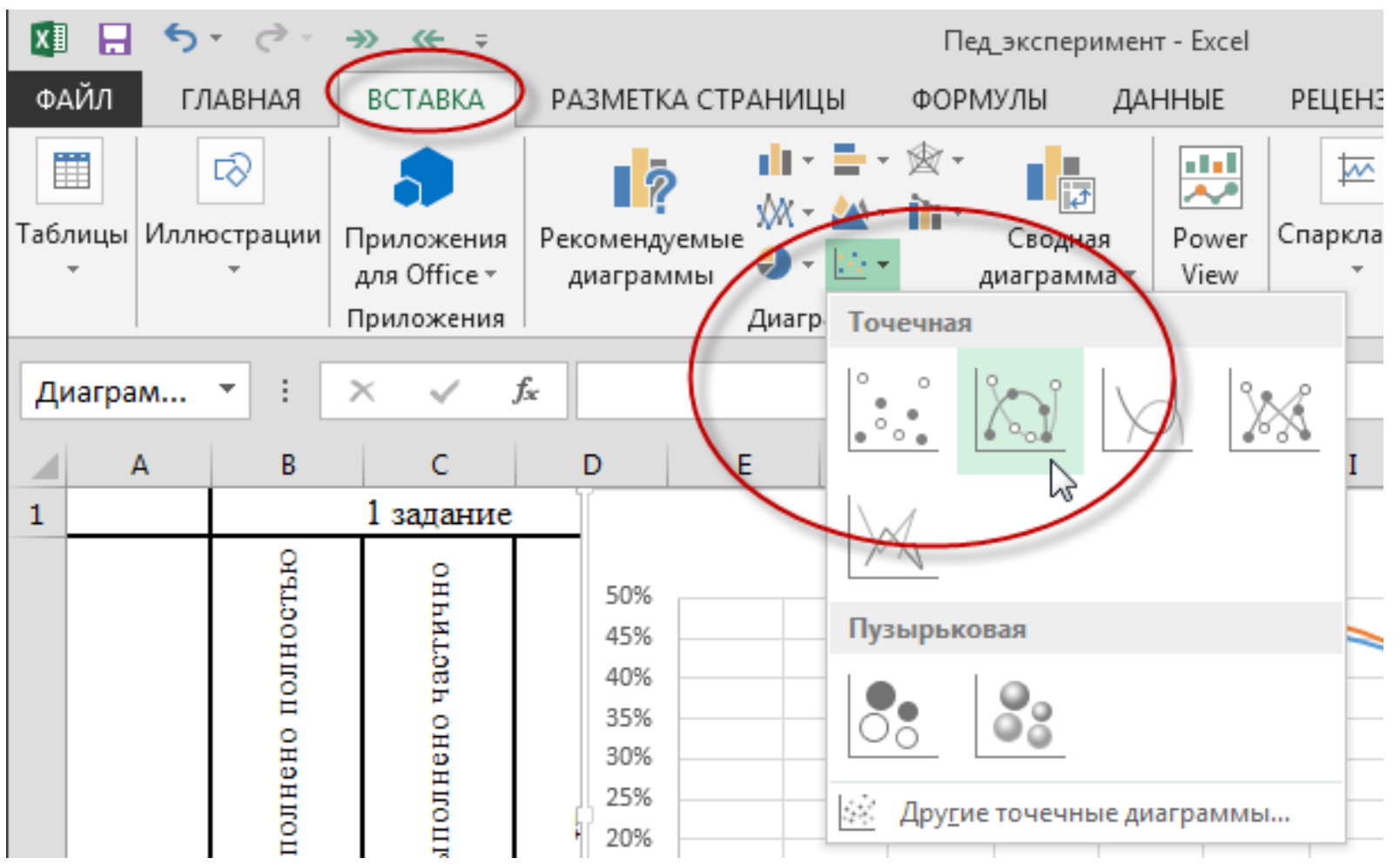

Рисунок 3 - Построение полигона частот в MS Excel.

В итоге вы получите полигоны частот, на основе которых можно предположить, что степень подготовленности в экспериментальной и контрольной группах одинакова.

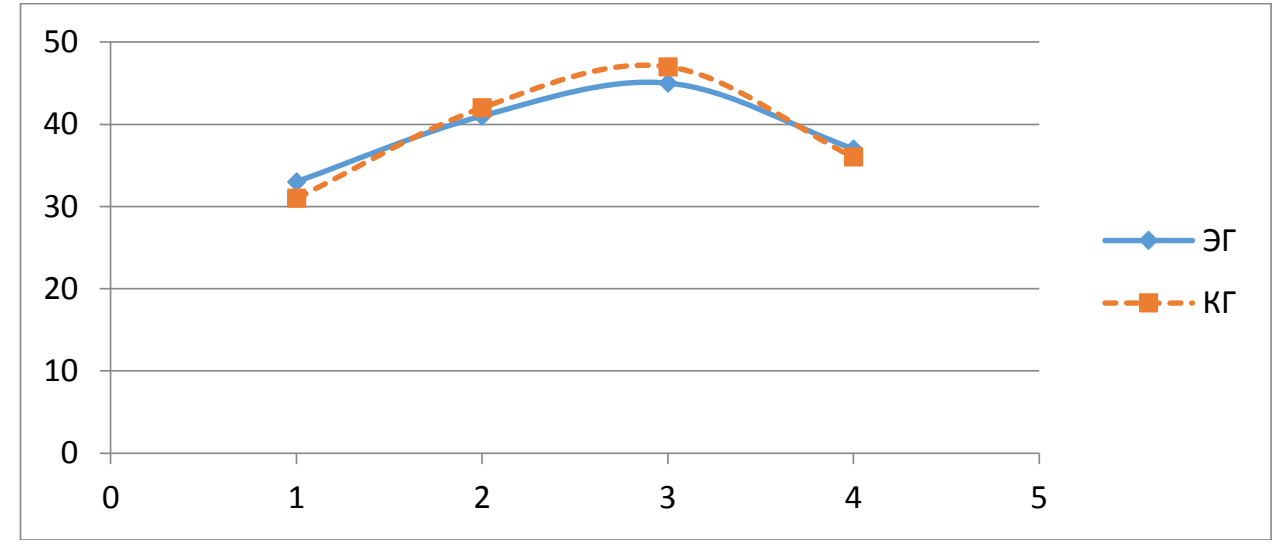

Рисунок 4 - Полигоны частот.

Измерения уровня знаний проводились после изучения каждого из разделов курса «Информатика и ИТ». В таблицах приведены результаты контролирующих лабораторных работ экспериментальных и контрольных групп.

В ходе педагогического эксперимента исследователь фиксирует результаты измерений уровня знаний в контрольной и экспериментальной группах до и после эксперимента и отображает их в виде таблиц и гистограмм. Для этого создаются два отдельных листа: До эксперимента и После эксперимента. На первом листе вводятся результаты эксперимента до проведения эксперимента в виде следующих таблиц: 


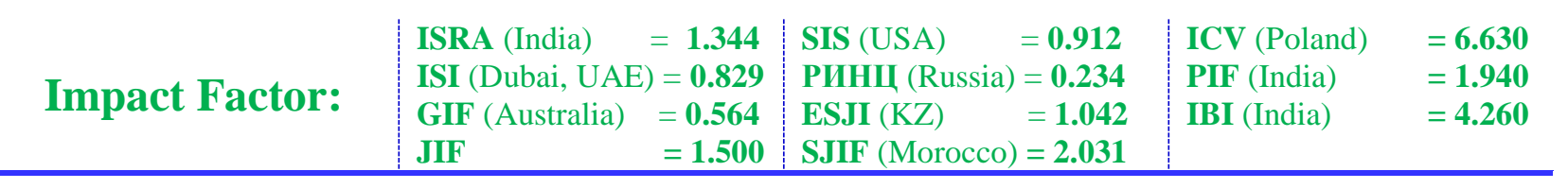

\begin{tabular}{|c|c|c|c|c|c|c|}
\hline$\Delta$ & A & B & c & D & $\mathrm{E}$ & $\mathrm{F}$ \\
\hline 1 & \multicolumn{6}{|c|}{ Результаты измерений уровня знаний в контрольной и экспериментальной группах до эксперимента } \\
\hline 2 & & & & & & \\
\hline 3 & & \multicolumn{4}{|c|}{ Критерии оценивания знаний } & \multirow{2}{*}{$\begin{array}{c}\text { Охват } \\
\text { студентов }\end{array}$} \\
\hline 4 & & Отлично & Хорошо & Удовл. & Неудовл. & \\
\hline 5 & Экспериментальная группа & 8 & 49 & 45 & 9 & 111 \\
\hline 6 & Контрольная группа & 10 & 42 & 43 & 9 & 104 \\
\hline \multicolumn{7}{|l|}{7} \\
\hline 8 & & \multicolumn{4}{|c|}{$\begin{array}{c}\text { Результаты оценивания знаний } \\
\text { (в \%) }\end{array}$} & \multirow{2}{*}{$\begin{array}{c}\text { Охват } \\
\text { студентов }\end{array}$} \\
\hline 9 & & Отлично & Хороmо & Удовл. & Неудовл. & \\
\hline 10 & Экспериментальная группа & $=\mathrm{B} 5 / \mathrm{SF} S 5$ & $=\mathrm{C} 5 / \mathrm{SF} S 5$ & $=\mathrm{D} 5 / \mathrm{SF} 55$ & $=\mathrm{E} 5 / \mathrm{SFS} 5$ & $\mathrm{n}_{1}=111$ \\
\hline 11 & Контрольная группа & $=\mathrm{B} 6 / \mathrm{SF}$ S 6 & $=\mathrm{C} 6 / \mathrm{SFS} 6$ & $=\mathrm{D} 6 / \mathrm{SF} 6$ & $=\mathrm{E} 6 / \mathrm{SFS} 6$ & $\mathrm{n}_{2}=104$ \\
\hline
\end{tabular}

Рисунок 5 - Подсчет результатов оценивания знаний в процентном соотношении в MS Excel.

Далее на основе процентных данных строится диаграмма: 


\begin{tabular}{l|lrl|l|ll} 
& ISRA (India) & $=\mathbf{1 . 3 4 4}$ & SIS (USA) & $=\mathbf{0 . 9 1 2}$ & ICV (Poland) & $=\mathbf{6 . 6 3 0}$ \\
Impact Factor: & ISI (Dubai, UAE) $=\mathbf{0 . 8 2 9}$ & PUHL (Russia) $=\mathbf{0 . 2 3 4}$ & PIF (India) & $=\mathbf{1 . 9 4 0}$ \\
& GIF (Australia) & $\mathbf{0 . 5 6 4}$ & ESJI (KZ) & $=\mathbf{1 . 0 4 2}$ & IBI (India) & $=\mathbf{4 . 2 6 0}$ \\
& JIF & $\mathbf{1 . 5 0 0}$ & SJIF (Morocco) & $=\mathbf{2 . 0 3 1}$ & & \\
\hline
\end{tabular}

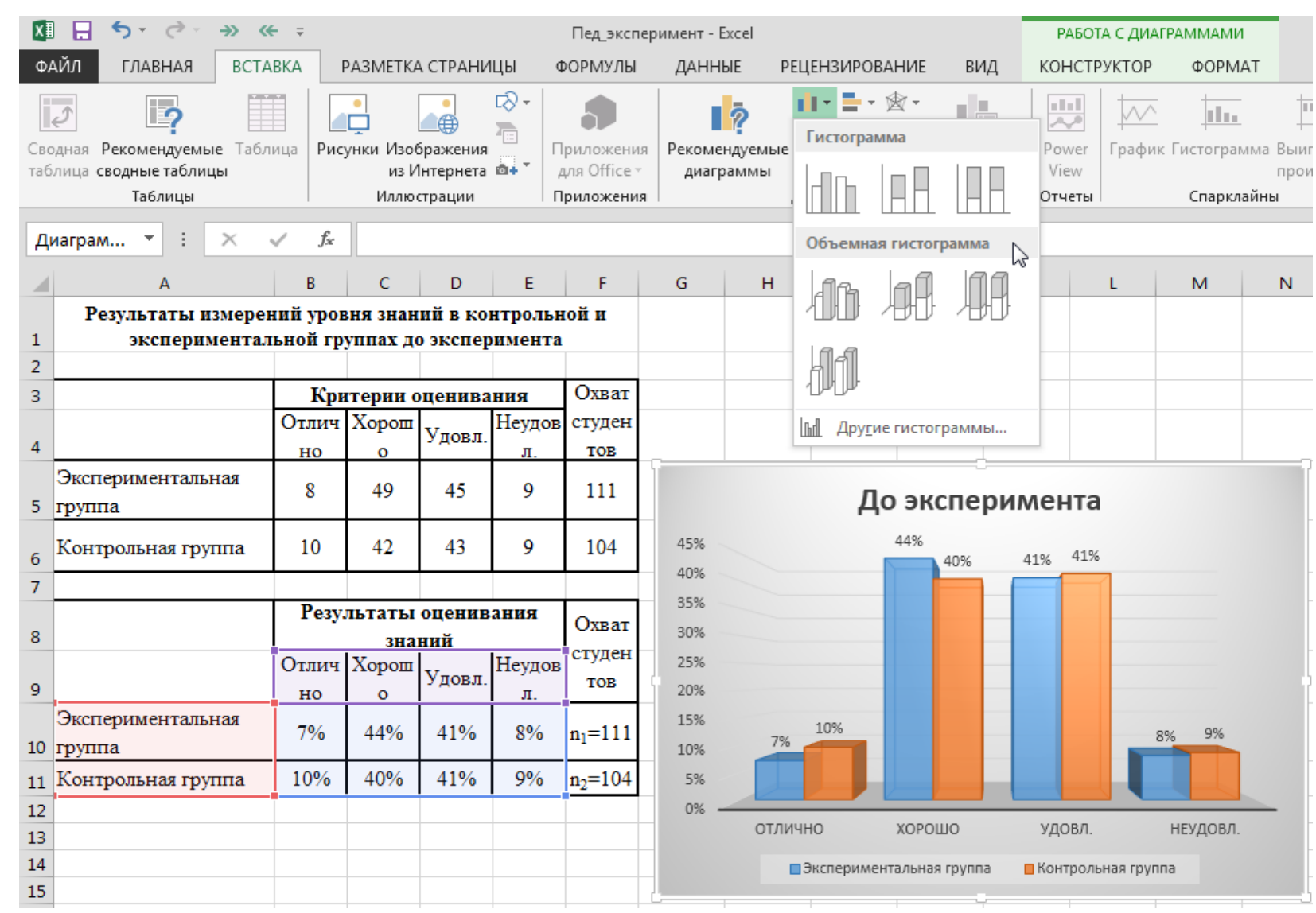

Рисунок 6 - Построение диаграммы по результатам измерений до начала эксперимента.

Аналогично вносятся результаты измерений уровня знаний в контрольной и экспериментальной группах после окончания

эксперимента и на их основе строится гистограмма:

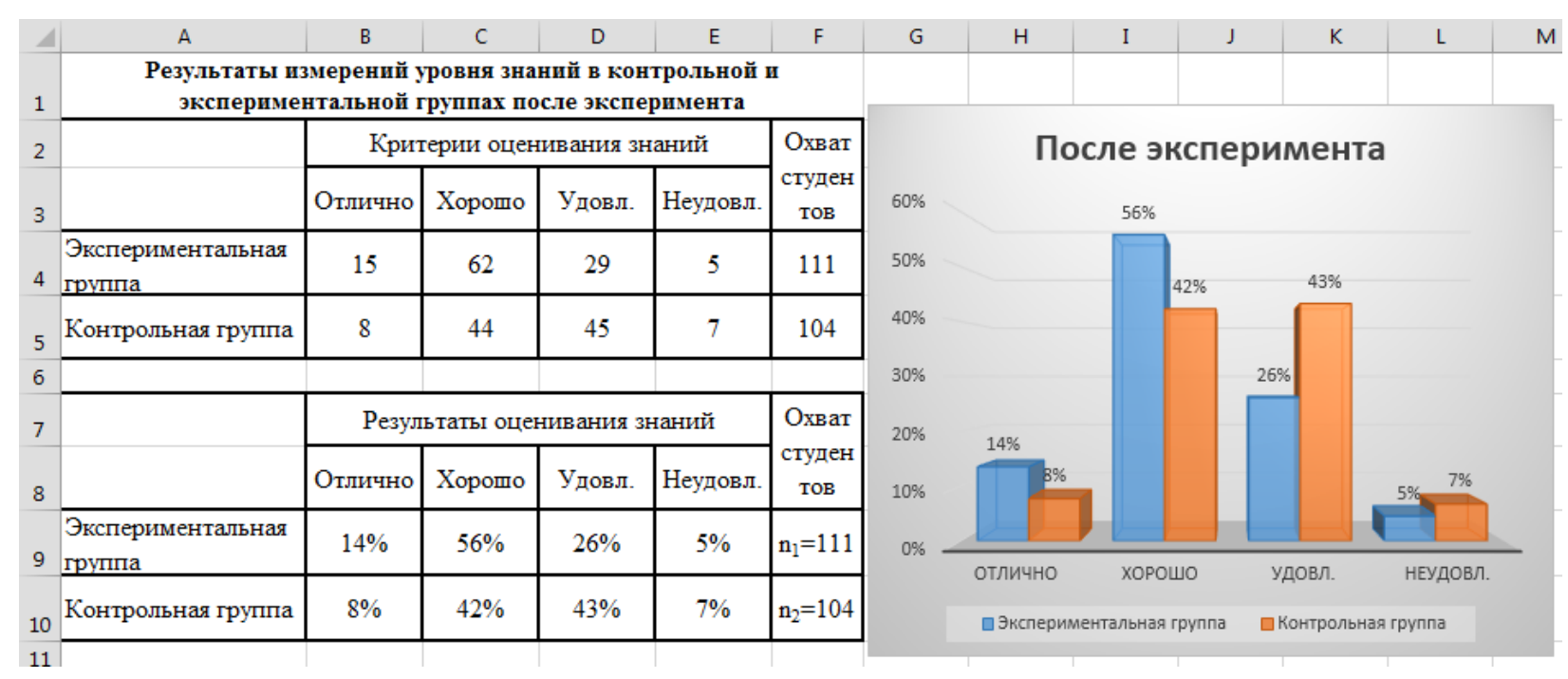

Рисунок 7 - Результаты измерений после эксперимента.

ISPC Global Applied Research,

Harrisburg, USA 


\begin{tabular}{|c|c|c|c|c|c|c|}
\hline Impact Factor: & $\begin{array}{l}\text { ISRA (India) } \\
\text { ISI (Dubai, UAF } \\
\text { GIF (Australia) } \\
\text { JIF }\end{array}$ & $\begin{array}{l}=1.344 \\
=0.829 \\
=0.564 \\
=1.500\end{array}$ & $\begin{array}{l}\text { SIS (USA) } \\
\text { PИНЦ (Russia) } \\
\text { ESJI (KZ) } \\
\text { SJIF (Morocco) }\end{array}$ & $\begin{array}{l}=0.912 \\
=0.234 \\
=1.042 \\
=2.031\end{array}$ & $\begin{array}{l}\text { ICV (Poland) } \\
\text { PIF (India) } \\
\text { IBI (India) }\end{array}$ & $\begin{array}{l}=6.630 \\
=1.940 \\
=4.260\end{array}$ \\
\hline
\end{tabular}

После проведения констатирующего, поискового и обучающего этапов педагогического эксперимента анализируются и обобщаются итоги экспериментальнотеоретического исследования.

Обозначим: $\mathrm{n}_{1}$ - количество студентов экспериментальной группы, $\mathrm{n}_{2}-$ количество студентов контрольной группы. С - количество градаций. $\mathrm{B}$ нашем случае $\mathrm{C}=4$ (неудовлетворительно, удовлетворительно, хорошо, отлично). $\mathrm{O}_{1 \mathrm{i}}-$ число членов экспериментальной группы, получивших і-ю категорию по состоянию изучаемого свойства; $\mathrm{O}_{2 \mathrm{i}}$ - число членов контрольной группы, получивших i-ю категорию по состоянию изучаемого свойства.

Результаты контролирующей лабораторной работы используем для проверки гипотезы о том, что обучаемые контрольной и

экспериментальной групп до начала эксперимента и после окончания эксперимента имеют равную вероятность попасть в одну из четырех категорий: неудовлетворительно, удовлетворительно, хорошо, отлично, т.е. проверить выполнение всех следующих равенств: $\mathrm{p}_{11}=\mathrm{p}_{21}, \mathrm{p}_{12}=\mathrm{p}_{22}, \mathrm{p}_{13}=\mathrm{p}_{23}, \mathrm{p}_{14}=\mathrm{p}_{24}$. Таким образом, нулевая гипотеза будет иметь вид $\mathrm{H}_{0}$ : $\mathrm{p}_{1 \mathrm{i}}$ $=\mathrm{p}_{2 \mathrm{i}}$. Альтернативная гипотеза будет иметь вид $\mathrm{H}_{1}: \mathrm{p}_{1 \mathrm{i}} \neq \mathrm{p}_{2 \mathrm{i}}$ хотя бы для одной из С категорий.

Обработка полученных данных производим методом $\chi^{2}$ - хи квадрат [9, С.101], вычисляемым по формуле:

$$
\mathrm{T}=\frac{1}{n_{1} \cdot n_{2}} \sum_{i=1}^{C} \frac{\left(n_{1} O_{2 i}-n_{2} O_{1 i}\right)^{2}}{O_{1 i}+O_{2 i}},
$$

где Т - эмпирическое значение.

Измерим значение Т для данных, полученных до начала эксперимента:

\begin{tabular}{|c|c|c|c|c|c|c|}
\hline$\Delta$ & A & B & C & D & $\mathrm{E}$ & $\mathrm{F}$ \\
\hline 1 & \multicolumn{6}{|c|}{ Вычисление эмпирического значения Т } \\
\hline 2 & & \multicolumn{4}{|c|}{ До эксперимента } & \\
\hline 3 & & $\mathrm{i}=1$ & $\mathrm{i}=2$ & $\mathrm{i}=3$ & $\mathrm{i}=4$ & \\
\hline 4 & $\mathrm{O}_{1 \mathrm{i}}$ & 8 & 49 & 45 & 9 & 111 \\
\hline 5 & $\mathrm{O}_{2 \mathrm{i}}$ & 10 & 42 & 43 & 9 & 104 \\
\hline 6 & $\mathrm{O}_{1 \mathrm{i}}+\mathrm{O}_{2 \mathrm{i}}=$ & 18 & 91 & 88 & 18 & \\
\hline 7 & $\mathrm{n} 1 * \mathrm{n} 2=$ & & & & & 11544 \\
\hline 8 & $\left(\mathrm{n}_{1} * \mathrm{O}_{2 \mathrm{i}}-\mathrm{n}_{2} * \mathrm{O}_{1 \mathrm{i}}\right)^{2} /(\mathrm{O} 1 \mathrm{i}+\mathrm{O} 2 \mathrm{i})=$ & 4293,56 & 2069,85 & 98,28 & 220,5 & \\
\hline 9 & $\mathrm{~T}=$ & 0,5788 & & & & \\
\hline
\end{tabular}

\section{Рисунок 8 - Вычисление эмпирического значения до эксперимента в MS Excel.}

Та же таблица с отображением всех рабочих

формул:

\begin{tabular}{|c|c|c|c|c|c|c|}
\hline$\Delta$ & A & B & c & D & E & $\mathrm{F}$ \\
\hline \multicolumn{7}{|c|}{ Вычисление эмпирического значения T } \\
\hline 2 & & \multicolumn{4}{|c|}{ До эксперимента } & Охват \\
\hline 3 & & $\mathrm{i}=1$ & $i=2$ & $i=3$ & $\mathrm{i}=4$ & тов \\
\hline & $\mathrm{O}_{1 \mathrm{i}}$ & 8 & 49 & 45 & 9 & 111 \\
\hline 5 & $\mathrm{O}_{2 \mathrm{i}}$ & 10 & 42 & 43 & 9 & 104 \\
\hline & $\mathrm{O}_{1}+\mathrm{O}_{2}=$ & $=\mathrm{B} 4+\mathrm{B} 5$ & $=\mathrm{C} 4+\mathrm{C} 5$ & $=\mathrm{D} 4+\mathrm{D} 5 \mathrm{~s}$ & $=E 4+E 5$ & \\
\hline & $\mathrm{n} 1 * \mathrm{n} 2=$ & & & & & $=F 4^{*} \times 5$ \\
\hline & $\left(\mathrm{n}_{1} * \mathrm{O}_{2 i}-\mathrm{n}_{2} * \mathrm{O}_{1 i}\right)^{2} /(\mathrm{O} 1 \mathrm{i}+\mathrm{O} 2 \mathrm{i})=$ & $=\mathrm{CTEMEHb}\left(\$ \mathrm{~F} \$ 4 * \mathrm{~B} 5-\$ \mathrm{SF} \$ 5^{*} \mathrm{~B} 4 ; 2\right) / \mathrm{B} 6$ & $=\mathrm{CTE} \Pi E H b\left(\$ F \$ 4^{*} \mathrm{C} 5-\$ F \$ 5 * \mathrm{C} 4 ; 2\right) / \mathrm{C} 6$ & f $=\mathrm{CTEMEHb}\left(\right.$ SF $\left.\$ 4 * \mathrm{D} 5-\mathrm{SF} \$ 5^{*} \mathrm{D} 4 ; 2\right) / \mathrm{D} 6$ & $6=\mathrm{CTE \Pi EHb}\left(\$ F \$ 4^{*} \mathrm{E} 5-\$ \mathrm{SF} S 5 * \mathrm{E} 4 ; 2\right) / \mathrm{E} 6$ & \\
\hline 9 & $T=$ & $=\mathrm{I} / \mathrm{F} 7 *(\mathrm{~B} 8+\mathrm{C} 8+\mathrm{D} 8+\mathrm{E} 8)$ & & & & \\
\hline
\end{tabular}

Рисунок 9 - Формулы для вычисления эмпирического значения. 0,5788 .

По таблице $[1$, С. 130$]$ для $\alpha=0,05$ и числа степеней свободы $v=\mathrm{C}-1=3$ находим критическое значение статистики критерия

ISPC Global Applied Research, 


\begin{tabular}{|c|c|c|c|c|c|c|}
\hline Impact Factor: & $\begin{array}{l}\text { ISRA (India) } \\
\text { ISI (Dubai, UAF } \\
\text { GIF (Australia) } \\
\text { JIF }\end{array}$ & $\begin{array}{l}=1.344 \\
=0.829 \\
=0.564 \\
=1.500\end{array}$ & $\begin{array}{l}\text { SIS (USA) } \\
\text { PИНЦ (Russia) } \\
\text { ESJI (KZ) } \\
\text { SJIF (Morocco) }\end{array}$ & $\begin{array}{l}=0.912 \\
=0.234 \\
=1.042 \\
=\mathbf{2 . 0 3 1}\end{array}$ & $\begin{array}{l}\text { ICV (Poland) } \\
\text { PIF (India) } \\
\text { IBI (India) }\end{array}$ & $\begin{array}{l}=6.630 \\
=1.940 \\
=4.260\end{array}$ \\
\hline
\end{tabular}

$\mathrm{T}_{\text {критич }}=7,815$. Отсюда верно равенство $\mathrm{T}_{\text {наблюд }}<$ $\mathrm{T}_{\text {критич }}(0,5788<7,815)$, т.е. в соответствии с правилом принятия решения для критерия $\chi^{2}$ полученный результат не дает достаточных оснований для отклонения нулевой гипотезы. Иначе говоря, контрольная и экспериментальная группы до начала эксперимента имели равную вероятность попасть в одну из четырех категорий: неудовлетворительно, удовлетворительно, хорошо, отлично.

Проверим нулевую гипотезу для данных, полученных после окончания эксперимента:

\begin{tabular}{|c|c|c|c|c|c|}
\hline \multicolumn{6}{|c|}{ Вычисление эмпирического значения Т } \\
\hline & \multicolumn{4}{|c|}{ После эксперимента } & \multirow{2}{*}{$\begin{array}{l}\text { Охват } \\
\text { студен } \\
\text { тов }\end{array}$} \\
\hline & $\mathrm{i}=1$ & $\mathrm{i}=2$ & $\mathrm{i}=3$ & $\mathrm{i}=4$ & \\
\hline $\mathrm{O}_{1 \mathrm{i}}$ & 15 & 62 & 29 & 5 & 111 \\
\hline $\mathrm{O}_{2 \mathrm{i}}$ & 8 & 44 & 45 & 7 & 104 \\
\hline $\mathrm{O}_{1 \mathrm{i}}+\mathrm{O}_{2 \mathrm{i}}=$ & 23 & 106 & 74 & 12 & \\
\hline $\mathrm{n} 1 * \mathrm{n} 2=$ & & & & & 11544 \\
\hline$\left(\mathrm{n}_{1} * \mathrm{O}_{2 \mathrm{i}}-\mathrm{n}_{2} * \mathrm{O}_{1 \mathrm{i}}\right)^{2} /(\mathrm{O} 1 \mathrm{i}+\mathrm{O} 2 \mathrm{i})=$ & 19634,09 & 23076,38 & 52924,88 & 5504,083333 & \\
\hline $\mathrm{T}=$ & 8,7612 & & & & \\
\hline
\end{tabular}

Рисунок 10 - Вычисление эмпирического значения после эксперимента в MS Excel.

Поскольку $\mathrm{T}_{\text {наблюд }}>\mathrm{T}_{\text {критич }}(8,7612>7,815)$, то нулевая гипотеза отклоняется на уровне $\alpha=0,05$ и принимается альтернативная гипотеза. Это значит, что при экспериментальном обучении различия в внаниях обучаемых экспериментальной группы по сравнению с контрольной являются статистически значимыми.

Осталось вычислить средний показатель успеваемости в контрольной и экспериментальной группах после окончания эксперимента:

$$
\begin{gathered}
\bar{x}_{\ni}=\frac{1}{n_{1}} \sum_{i=1}^{4} \mathrm{O}_{1 i} x_{i}=\frac{1}{111}(15 * 5+62 * 4+29 * 3+5 * 2)=3,78 ; \\
\bar{x}_{\mathrm{K}}=\frac{1}{n_{2}} \sum_{i=1}^{4} \mathrm{O}_{2 i} x_{i}=\frac{1}{104}(8 * 5+44 * 4+45 * 3+7 * 2)=3,51 .
\end{gathered}
$$

\begin{tabular}{|c|c|c|c|c|c|c|}
\hline 4 & A & B & $\mathrm{C}$ & D & $\mathrm{E}$ & $\mathrm{F}$ \\
\hline 1 & \multicolumn{6}{|c|}{ Средние показатели успеваемости } \\
\hline 2 & & \multicolumn{4}{|c|}{ После эксперимента } & Oxват \\
\hline 3 & & 5 & 4 & 3 & 2 & $\begin{array}{c}\text { студен } \\
\text { тов }\end{array}$ \\
\hline 4 & $\mathrm{O}_{1 \mathrm{i}}$ & 15 & 62 & 29 & 5 & 111 \\
\hline 5 & $\mathrm{O}_{2 \mathrm{i}}$ & 8 & 44 & 45 & 7 & 104 \\
\hline 6 & $\mathrm{O}_{11}{ }^{*} \mathrm{x}_{1}=$ & 75 & 248 & 87 & 10 & \\
\hline 7 & $\mathrm{O}_{2 i}{ }^{*} \mathrm{x}_{1}=$ & 40 & 176 & 135 & 14 & \\
\hline 8 & $\begin{array}{l}\text { Ср.показатель успеваемости } \\
\text { в экспериментальной группе } \\
\left(x_{。}\right)\end{array}$ & 3,78 & \multirow{2}{*}{$\begin{array}{l}\text { Соотнопение } \\
\mathrm{x}_{y} / \mathrm{x}_{\mathrm{x}}\end{array}$} & \multirow{2}{*}{1,08} & & \\
\hline 9 & $\begin{array}{l}\text { Ср.показатель успеваемости } \\
\text { в контрольной группе }\left(\mathrm{x}_{\mathrm{k}}\right)\end{array}$ & $\mathbf{3 , 5 1}$ & & & & \\
\hline
\end{tabular}

Рисунок 11 - Вычисление средних показателей успеваемости в MS Excel.

Эта же таблица в формулах:

ISPC Global Applied Research, 


\begin{tabular}{|c|c|c|c|c|c|c|}
\hline Impact Factor: & $\begin{array}{l}\text { ISRA (India) } \\
\text { ISI (Dubai, UAF } \\
\text { GIF (Australia) } \\
\text { JIF }\end{array}$ & $\begin{array}{l}=1.344 \\
=0.829 \\
=0.564 \\
=1.500\end{array}$ & $\begin{array}{l}\text { SIS (USA) } \\
\text { PИНЦ (Russia) } \\
\text { ESJI (KZ) } \\
\text { SJIF (Morocco) }\end{array}$ & $\begin{array}{l}=0.912 \\
=0.234 \\
=1.042 \\
=2.031\end{array}$ & $\begin{array}{l}\text { ICV (Poland) } \\
\text { PIF (India) } \\
\text { IBI (India) }\end{array}$ & $\begin{array}{l}=6.630 \\
=1.940 \\
=4.260\end{array}$ \\
\hline
\end{tabular}

\begin{tabular}{|c|c|c|c|c|c|c|}
\hline 4 & A & B & c & D & E & $\mathrm{F}$ \\
\hline 1 & & \multicolumn{5}{|c|}{ Средние показатели успеваемости } \\
\hline 2 & & \multicolumn{4}{|c|}{ После эксперимента } & Охват \\
\hline 3 & & 5 & 4 & 3 & 2 & студентов \\
\hline 4 & $\mathrm{O}_{1 \mathrm{i}}$ & 15 & 62 & 29 & 5 & 111 \\
\hline 5 & $\mathrm{O}_{2 \mathrm{i}}$ & 8 & 44 & 45 & 7 & 104 \\
\hline 6 & $\mathrm{O}_{11}{ }^{*} \mathrm{x}_{1}=$ & $=\mathrm{B} 4 * \$ \mathrm{~B} \$ 3$ & $=\mathrm{C} 4 * \$ \mathrm{C} \$ 3$ & $=\mathrm{D} 4 * \mathrm{D} \$ 3$ & $=E 4 * \$ E \$ 3$ & \\
\hline 7 . & $\mathrm{O}_{2 i}{ }^{*} \mathrm{x}_{1}=$ & $=\mathrm{B} 5 * \$ \mathrm{~B} \$ 3$ & $=\mathrm{C} 5 * \$ \mathrm{C} \$ 3$ & $=\mathrm{D} 5 * \$ \mathrm{D} \$ 3$ & $=E 5 * \$ E \$ 3$ & \\
\hline 8 & $\begin{array}{l}\text { Ср.показатель успеваемости в } \\
\text { экспериментальной группе (x, }\end{array}$ & $=1 / F 4 * \mathrm{CYMM}(\mathrm{B} 6: \mathrm{E} 6)$ & Соотношение & & & \\
\hline 9 & $\begin{array}{l}\text { Ср.показатель успеваемости в } \\
\text { контрольной группе }\left(\mathrm{x}_{\mathrm{k}}\right)\end{array}$ & $=1 /$ F5*CУMM(B7:E7) & $\mathrm{x}_{\mathrm{g}} / \mathrm{x}_{\mathrm{K}}$ & $=\mathbf{B 8} / \mathbf{B} 9$ & & \\
\hline
\end{tabular}

Рисунок 12 - Формулы для вычисления средних показателей успеваемости.

\section{Conclusion}

Как видим, соотношение $\frac{\bar{x}_{9}}{\bar{x}_{\mathrm{K}}}$ дает значение 1,08, что позволяет утверждать, что эффективность обучения в экспериментальной группе по сравнению с контрольной группой увеличилась на $8 \%$. Следовательно, можно сделать вывод, что эффект изменений обусловлен именно применением экспериментальной методики обучения.

Таким образом, на этапе сбора и обработки данных педагогического исследования компьютер сегодня можно считать незаменимым. Он в значительной мере облегчает работу исследователя по регистрации, сортировке, хранению и переработке больших объёмов информации, полученных в ходе эксперимента, наблюдения, бесед, интервью, анкетирования и других методов исследовательской работы. Это позволяет исследователю сэкономить время, избежать ошибок при расчётах и сделать объективные и достоверные выводы из экспериментальной части работы.

\section{References:}

1. (2004) Kontseptsiya informatizatsii sfery obrazovaniya Respubliki Uzbekistan // Gazeta «Uchitel' Uzbekistana». - 9-16 april 2004.

2. Geyn AG (2000) Izuchenie informatsionnogo modelirovaniya kak sredstvo realizatsii mezhpredmetnykh svyazey informatiki $\mathrm{s}$ distsiplinami estestvennonauchnogo tsikla : avtoref. ... d-ra ped. nauk. - Moscow, 2000. $48 \mathrm{p}$.

3. Zverev ID, Maksimova VN (1981) Mezhpredmetnye svyazi v sovremennoy shkole. - Moscow: Pedagogika, 1981. - 159 p.

4. Kachanov AN (2003) Mezhpredmetnye svyazi $\mathrm{v}$ protsesse prepodavaniya informatiki $\mathrm{v}$ turistskom vuze : dis... kand. ped. nauk: 13.00.08. - Moscow, 2003. -105 p.
5. Makhmutov MI, Shakirzyanov AZ (1985) Uchebnyy protsess $\mathrm{s}$ ispol'zovaniem mezhpredmetnykh svyazey $\mathrm{v}$ srednikh PTU : metod. posobie. - Moscow: Vysshaya shkola, 1985. $-207 \mathrm{p}$.

6. (2001) Gosudarstvennyy obrazovatel'nyy standart Uzbekistana. Trebovaniya $\mathrm{k}$ neobkhodimomu soderzhaniyu i urovnyu podgotovlennosti bakalavra po napravleniyu 5141300 - Rodnoy yazyk i literatura (Russkiy yazyk i literatura). - Tashkent, 2001. $-31 \mathrm{p}$.

7. Safonov VI (2008) Organizatsiya podgotovki uchiteley matematiki $\mathrm{k}$ ispol'zovaniyu informatsionnykh tekhnologiy // Kazanskiy pedagogicheskiy zhurnal. - 2008. - №2. pp.98-104. 


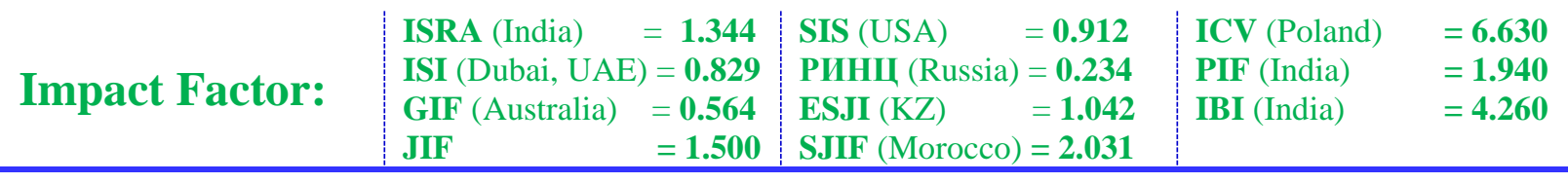

8. Nabiulina LM (2010) Mezhpredmetnye svyazi pri planirovanii laboratornykh rabot po informatike i informatsionnym tekhnologiyam $\mathrm{v}$ VUZakh Uzbekistana // zhurnal «Chelovek i obrazovanie» - 2010 №2 - pp.144-147

9. Grabar' MI, Krasnyanskaya KA (1977) Primenenie matematicheskoy statistiki V pedagogicheskikh issledovaniyakh.
Neparametricheskie metody. Moscow, «Pedagogika», 1977. - 136 p.

10. Sheraliyev S, Tigay O, Abdurakhmanova S, Alibekov S (2016) INTEGRATED TECHNIQUE FOR SOLVING PROBLEMS IN PHYSICS USING MATHCAD TOOLKIT AND CROCODILE TECHNOLOGY 3D. ISJ Theoretical \& Applied Science, 04 (36): 101105. 